\title{
A truly inclusive event
}

The British Dental Conference and

Dentistry Show 2022 will offer a truly inclusive event for all members of the dental team, regardless of previous experience, specialisms, career ambitions or roles.

Lecture programmes and interactive sessions will be available for all team members from dentists and dental nurses to dental hygienists and therapists, practice managers and treatment coordinators. You'll be able

\section{British Dental Conference \&}

Dentistry

\section{Show}

\section{Birminģ}

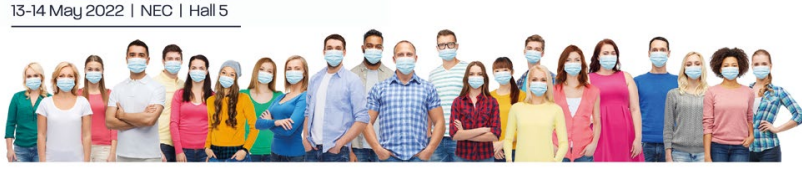

to advance clinical skills, broaden your experience and develop your knowledge on a wide range of business and administrative matters. Plus, gain verifiable $\mathrm{CPD}$, browse the latest innovations on the market and network with thousands of professionals from all corners of the industry.

Everyone's invited and it's set to be one heck of a show! Don't miss out. The next British Dental Conference and Dentistry Show will be held on Friday 13 and Saturday 14 May 2022, Birmingham NEC, co-located with DTS. For more information, visit www. thedentistryshow. co.uk.

\section{Supporting optimal infection control}

Enhance the treatment experience for patients with the cutting-edge Primescan intraoral scanner from Clark Dental. It is equipped with a Smart Pixel Sensor and dynamic depth scan technology, enabling it to capture high-quality images within seconds.

Primescan is also optimised to support your infection control protocols. It features a compact Acquisition Centre with smooth, easy-to-clean surfaces, and a sleek touch interface that facilitates improved standards of decontamination compared to a traditional keyboard.

Additionally, Primescan offers a choice of hygienic sleeves, including singleuse sleeves, an autoclavable sleeve with a disposable window, and a stainlesssteel sleeve that can be effectively wipe disinfected or dry heat sterilised.

For more information call Clark Dental on 01268733 146, email info@clarkdental. co.uk or visit www.clarkdental.co.uk.

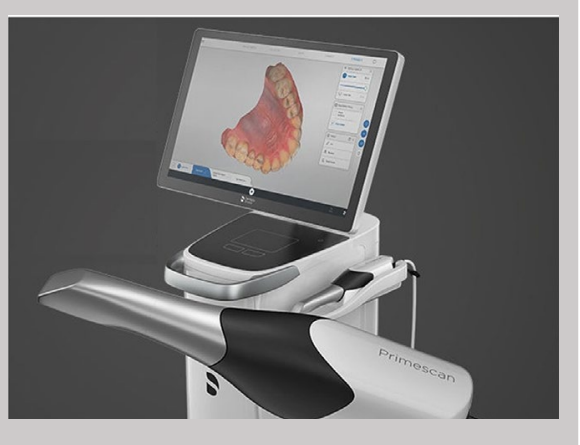

\section{Elevated oral health is a}

\section{lifestyle choice}

An effective oral care routine is central to achieving good health and wellbeing.

For patients who are making changes to pursue a better lifestyle, recommend the range of innovative solutions from leading oral healthcare specialist, Curaprox.

These include its Hydrosonic Easy electric toothbrush. This has a powerful hydrodynamic effect to deliver a thorough yet gentle clean to even hard-to-reach areas.

Comfortable to hold and manipulate around the mouth and gums, it will remove optimal bacteria, mitigating the damage caused by harmful acids.

A really clean mouth will make your patients feel good about themselves. For more information call 01480 862084, email info@curaprox.co.uk or visit www.curaprox.co.uk.

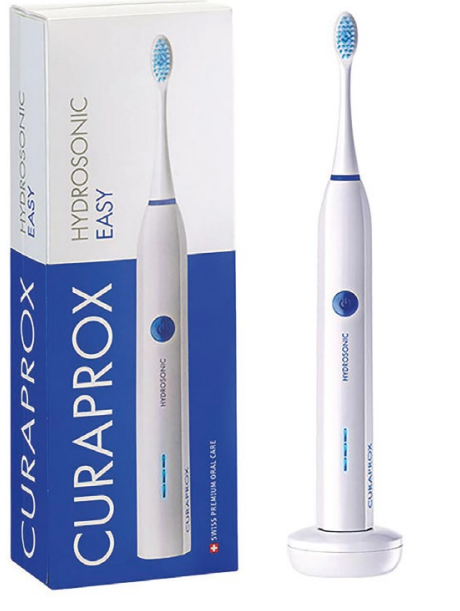

\section{The easy way to refer!}

When you need to refer a patient, you want the process to be simple. At EndoCare, this simplicity is key to our referral service! If you have a patient with complex endodontic needs, or perhaps a case where an individual is more anxious than usual as they are afraid of potential pain, you can refer them to us and we will guarantee to give them the care they need.

Over the years we have saved tens

of thousands of teeth that would have otherwise needed extraction, and because we only use the most cutting-edge techniques and products, treatment is always as pain-free as possible.

Following treatment, we always return patients to your care with comprehensive treatment notes and our suggested aftercare tips and next steps.

To find out more contact EndoCare on 02072240999 or visit www.endocare.co.uk.

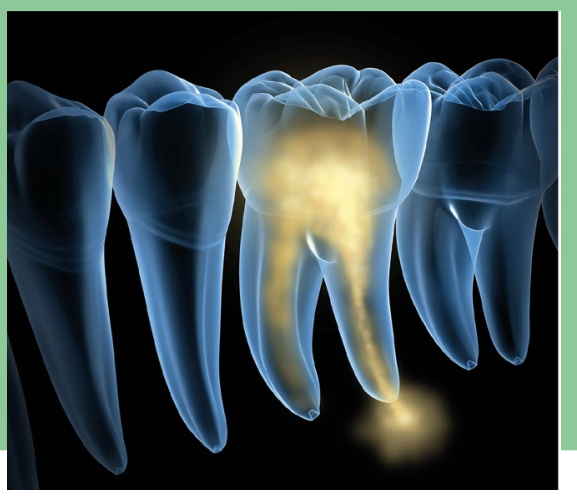

BDJ IN PRACTICE | VOL 34 | ISSUE 7 